\title{
Altered water renal handling in patients with HIV infection
}

\author{
Carlos Musso • Vincenzo Bellizzi · Waldo Belloso
}

Received: 22 October 2013/Accepted: 3 November 2013/Published online: 7 December 2013

(C) Italian Society of Nephrology 2013

Dear Editor,

I would like to comment on the excellent review regarding "management of hyponatremia in HIV disease" by Menon and colleagues that has been recently published in your journal [1]. In this article, the authors analyzed the conditions and drugs that may induce hyponatremia in patients with chronic HIV infection, such as: SIADH secondary to opportunistic lung (or cerebral) infections or neoplastic invasion, cerebral salt wasting syndrome, hormonal disorders as well as different sort of drugs. We would like to comment that in our renal clinical physiology unit, we originally evaluated the renal water handling capability in clinically stable HIV-1 infected individuals (not suffering from AIDS) who had normal renal function and serum sodium levels, and neither receiving drugs that may induce hyponatremia nor being affected by any sort of further infection or cancer disease [2,3]. In this evaluation, we found that HIV + patients were not able to neither adequately concentrate (urine osmolarity: HIV: $520 \pm 150$ mOsm/l vs. healthy controls: $900 \pm 100 \mathrm{mOsm} / \mathrm{l}$ ) nor

A response to this comment can be found at doi:10.1007/s40620-0130005-4.

\section{Musso ( $)$}

Nephrology Division, Hospital Italiano de Buenos, Aires, Argentina

e-mail: carlos.musso@hospitalitaliano.org.ar

\section{Bellizzi}

Division of Nephrology, University Hospital "San Giovanni di Dio e Ruggi d'Aragona”, Salerno, Italy

W. Belloso

Infectious Diseases Section of Internal Medicine Division,

Hospital Italiano de Buenos, Aires, Argentina dilute (urine osmolarity: HIV: $170 \pm 18 \mathrm{mOsm} / \mathrm{l}$ vs. healthy controls: $40 \pm 10 \mathrm{mOsm} / \mathrm{l}$ ) urine in a setting of a water restriction (12-h water restriction test) and water overload (Chaimowitz's test), respectively. Moreover, all these findings were consistent regardless of the presence or not of anti-retroviral therapy (either including tenofovir disoproxil fumarate or not). Regarding the mechanisms that could explain this abnormality in renal water handling, a severe reduction in their free water clearance $(3.5 \pm 3 \mathrm{ml} /$ $\min / 1.73 \mathrm{~m}^{2}$ ) was documented, although some influence of inappropriate vasopressin secretion could not be completely discarded. In conclusion, our findings indicate that in addition to the causes of hyponatremia commented in the review by Menon et al. there is an increased risk for dehydration or hyponatremia in clinically stable patients with chronic HIV-1 infection undergoing mild water deprivation or mild water overload, respectively, which may be aggravated in the context of receiving drugs or suffering from clinical settings that could potentially cause dysnatremia.

\section{References}

1. Menon M, Garcha A, Khanna A (2013) The management of hyponatremia in HIV disease. JNephrol 26(01):61-72

2. Musso CG, Navarro M, de Paz Sierra M, Perelsztein A, Sanchez M, Belloso W (2011) Severe alteration of free water clearance in HIV patients. In: 10th annual prevention in renal disease conference, Toronto (syllabus)

3. Musso CG, Navarro M, de Paz Sierra M, et al (2013) Proximal tubule function and free water clearance: comparison between healthy elderly and young HIV + patients. J Aging Sci 1:2. doi:10.4172/2329-8847.1000106 